\title{
Controlling neuronal cell growth through composite laminin supramolecular hydrogels
}

\author{
Rashmi Jain, ${ }^{\text {a Sangita Roy }}{ }^{\mathbf{a}^{*}}$ \\ Institute of Nano Science and Technology, Mohali, Punjab \\ Supporting Information
}

1. Table S1: Fiber diameter measurements of laminin derived hydrogels through AFM and TEM

2. Figure S1: HPLC- MS spectra of Fmoc IKVAV and Fmoc YIGSR

3. Figure S2: Calibration curve of Fmoc IKVAV and Fmoc YIGSR

4. Figure S3: Rheological measuements of IKVAV peptide with different hydrophobic modifications

5. Figure S4: TEM images of laminin derived individual peptide gels and composite selfsorted gel

6. Figure S5: Magnified TEM image of composite gel

7. Figure S6: Time dependent AFM imaging to demonstrate mixing behaviour of individual peptides in composite gels

8. Figure S7: Concentration dependent rheological measurements

9. Figure S8: Rhelogical analysis depicting thixotropic nature of laminin derived individual peptide gels and composite self-sorted gel

10. Figure S9: Optical images and atomic force microscopic images of deformed and reformed gels during thixotropic measurements

11. Figure S10: Correlation of CD signals with changing concentration ratio of peptides in composite gels

12. Figure S11: Cytotoxicity studies of laminin derived peptide hydrogels with $\mathrm{C} 6$ and SHSY5Y cells after 24 and $72 \mathrm{hrs}$

13. Figure S12: Microscopic images of 2D culture of C6 cells upto 5 days, over laminin hydrogels

14. Figure S13: Microscopic images of 2D culture of SHSY5Ycells upto 5 days, over laminin hydrogels

15. Figure S14: Immunofluorescence staining of neuronal marker $\beta$-III tubulin expressed in C6 cells cultured over laminin hydrogels

16. Figure S15: Immunofluorescence staining of neuronal marker $\beta$-III tubulin expressed in SHSY5Y cells cultured over laminin hydrogels

17. Figure S16: Cell cycle analysis of C6 and SHSY5Y cells treated with individual as well as conjugate laminin peptides 
Table S1: Fiber diameters of different laminin derived peptide hydrogels measured through AFM and TEM analysis through Image $J$ software.

\begin{tabular}{|c|c|c|}
\hline Hydrogel composition & Diameter by AFM (nm) & Diameter by TEM (nm) \\
\hline Fmoc IKVAV & $30 \pm 5 \mathrm{~nm}$ & $15 \pm 2.2 \mathrm{~nm}$ \\
\hline Fmoc YIGSR & $20 \pm 6 \mathrm{~nm}$ & $11.5 \pm 1.2 \mathrm{~nm}$ \\
\hline Fmoc IKVAV+Fmoc YIGSR & $85 \pm 14 \mathrm{~nm}$ and $32 \pm 6.5 \mathrm{~nm}$ & $28.3 \pm 2.5 \mathrm{~nm}$ and $9.5 \pm 1.9 \mathrm{~nm}$ \\
\hline
\end{tabular}



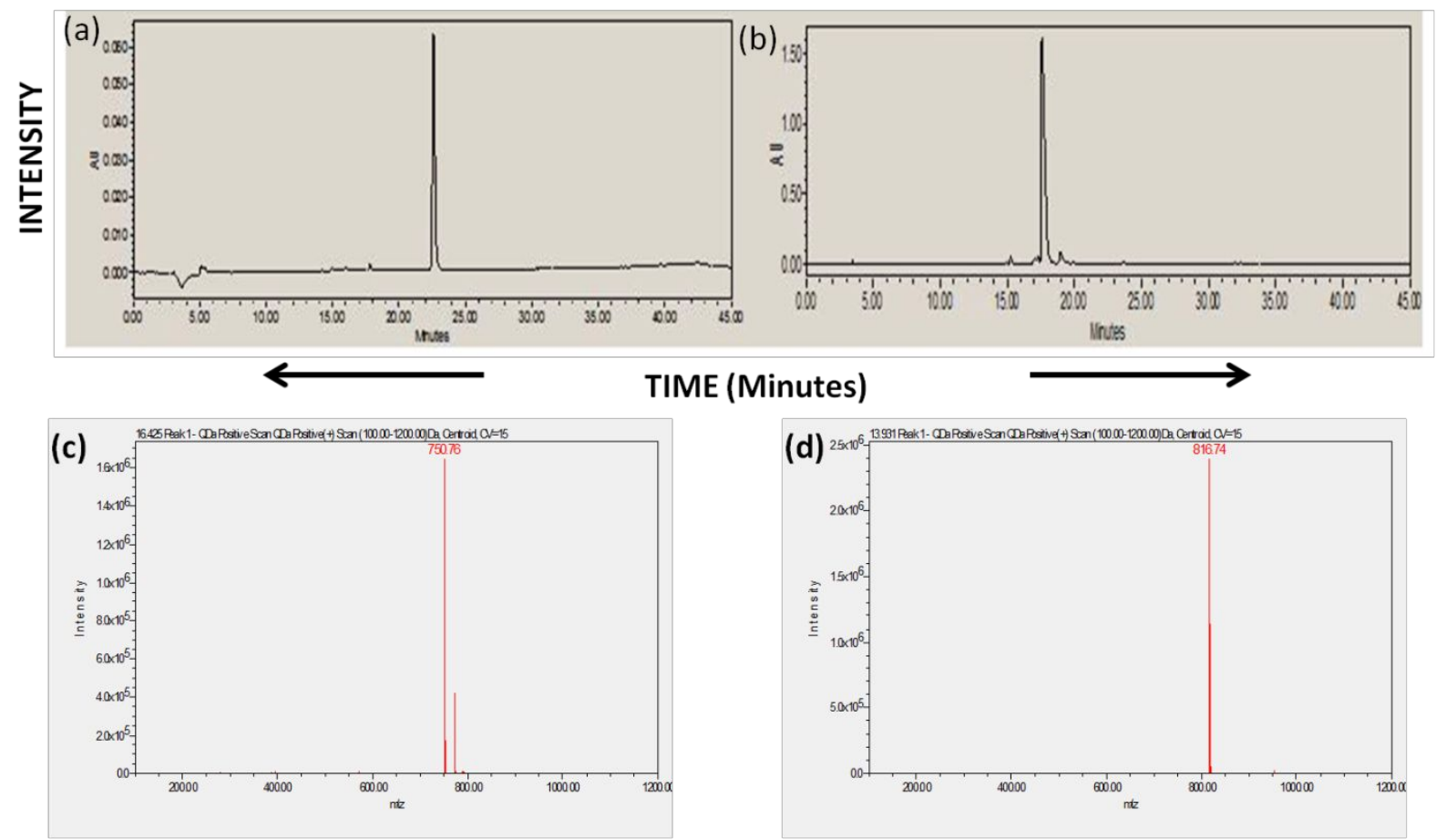

Figure S1: HPLC chromatograms and mass spectra of (a \& c) Fmoc IKVAV and (b \& d) Fmoc YIGSR. 

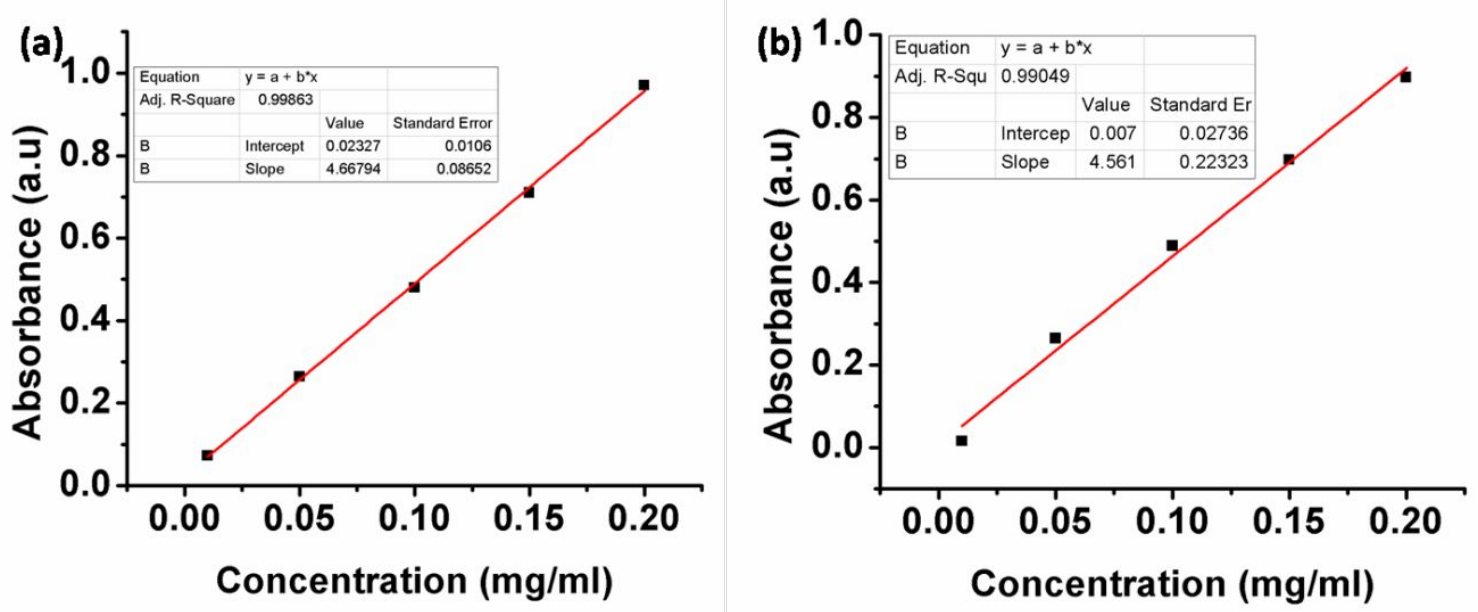

Figure S2: Calibration curve of (a) Fmoc IKVAV and (b) Fmoc YIGSR. 

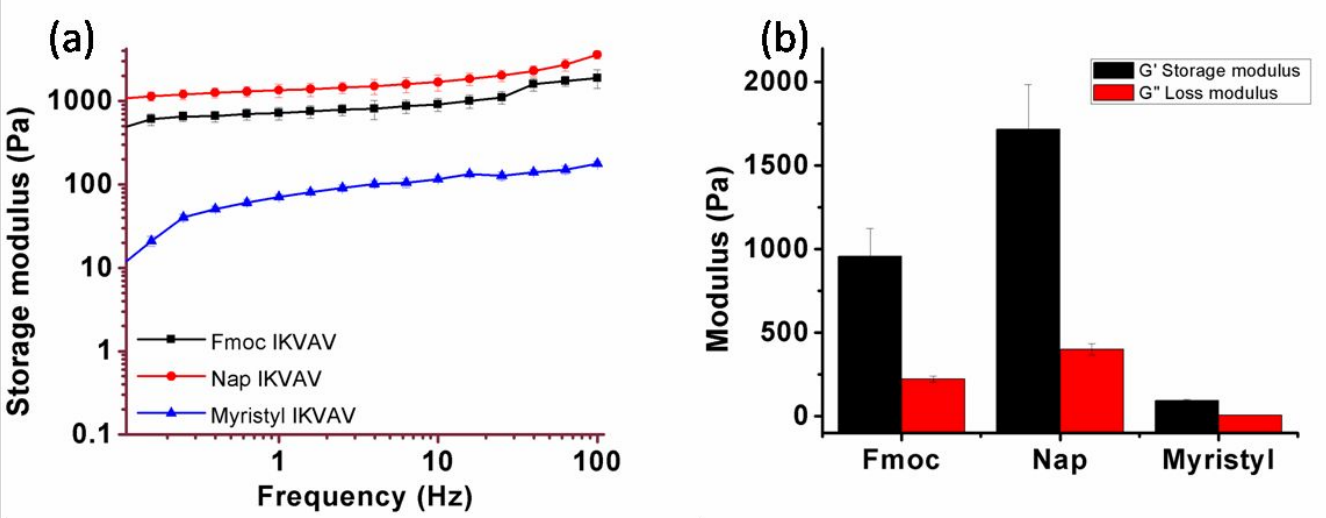

Figure S3: Rheological measurements of IKVAV peptide with different N-terminal modifications with Fmoc, Nap and Myristyl (a) showing change in storage modulus with respect to frequency for the hydrophobically modified IKVAV peptides and (b) comparing the average storage and loss modulus of each peptide. 

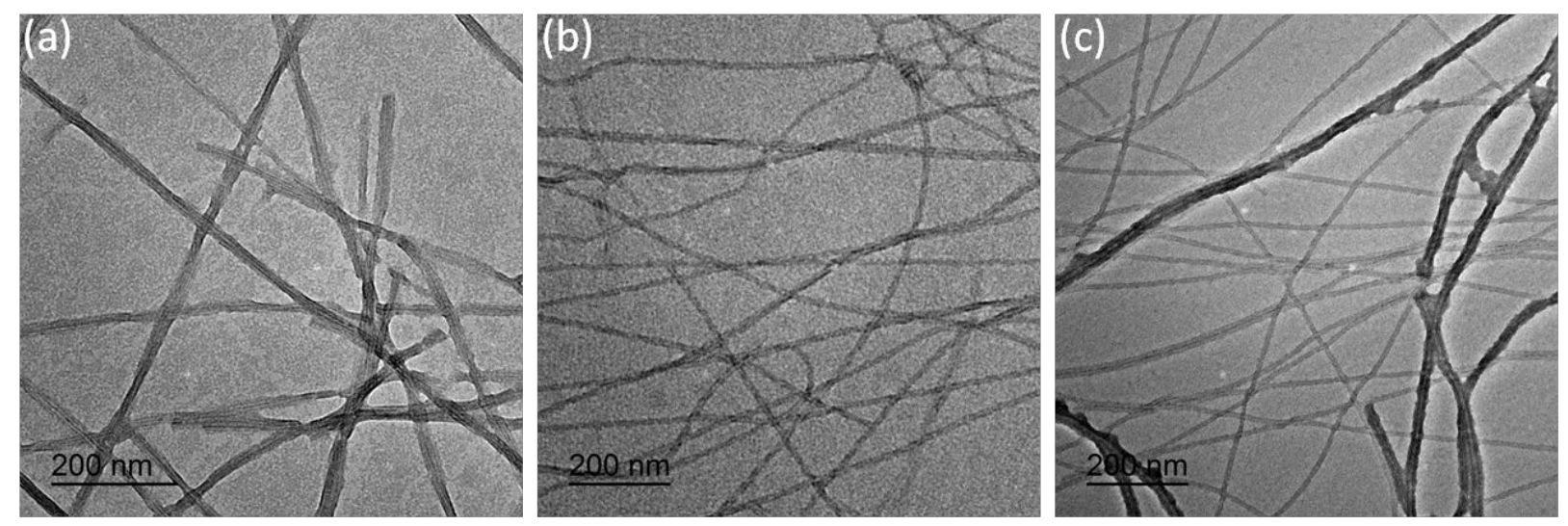

Figure S 4: TEM images of laminin derived peptides showing differential fibrous morphology formed by (a) Fmoc IKVAV, (b) Fmoc YIGSR and (c) combined self-sorted network of Fmoc IKVAV + Fmoc YIGSR.

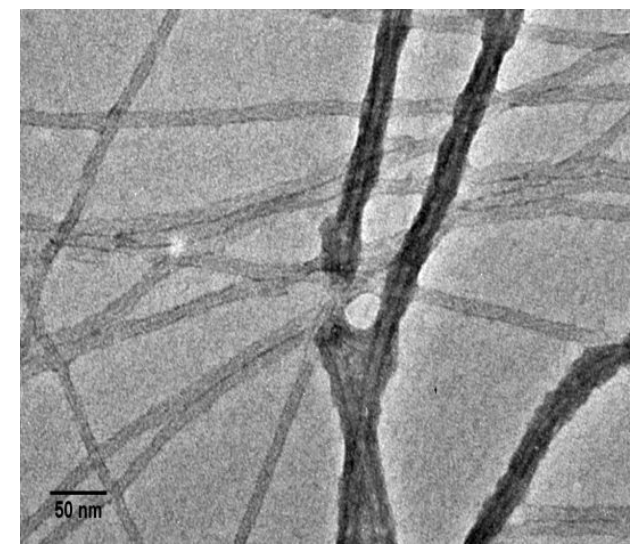

Figure S5: Magnified TEM image of Fmoc IKVAV and Fmoc YIGSR composite gel. 
(a)

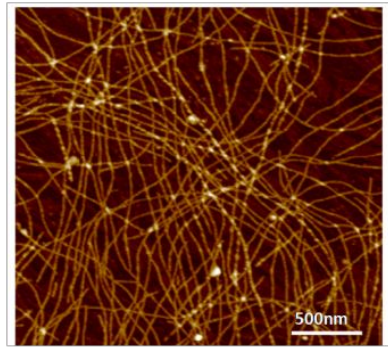

(d)

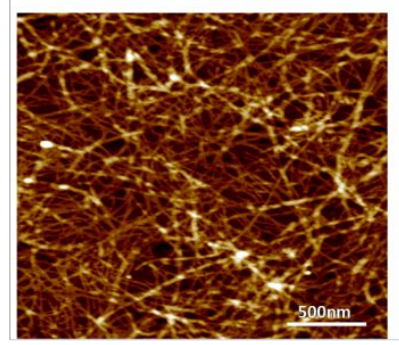

(b)
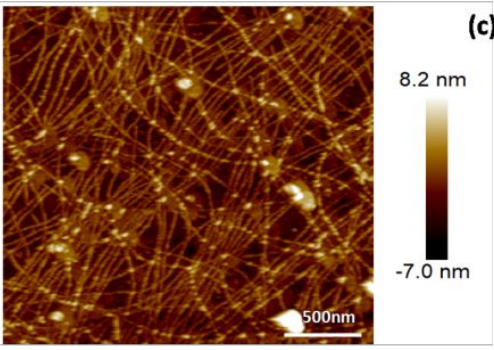

(e)

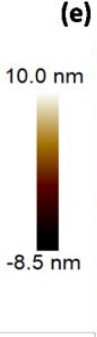

(c)

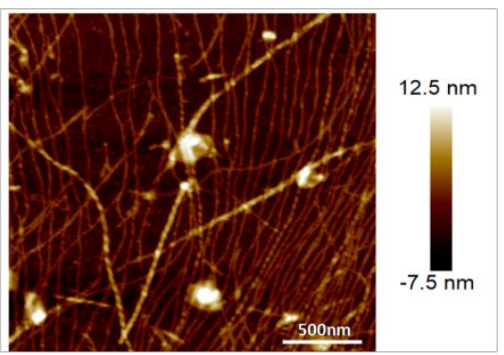

Figure S6: AFM images of composite gels showing self-assembling behavior of individual peptides in mixture at different time points (a) $5 \mathrm{~min}$, (b) $10 \mathrm{~min}$, (c) $15 \mathrm{~min}$, (d) $30 \mathrm{~min}$ and (e) 60 min. 

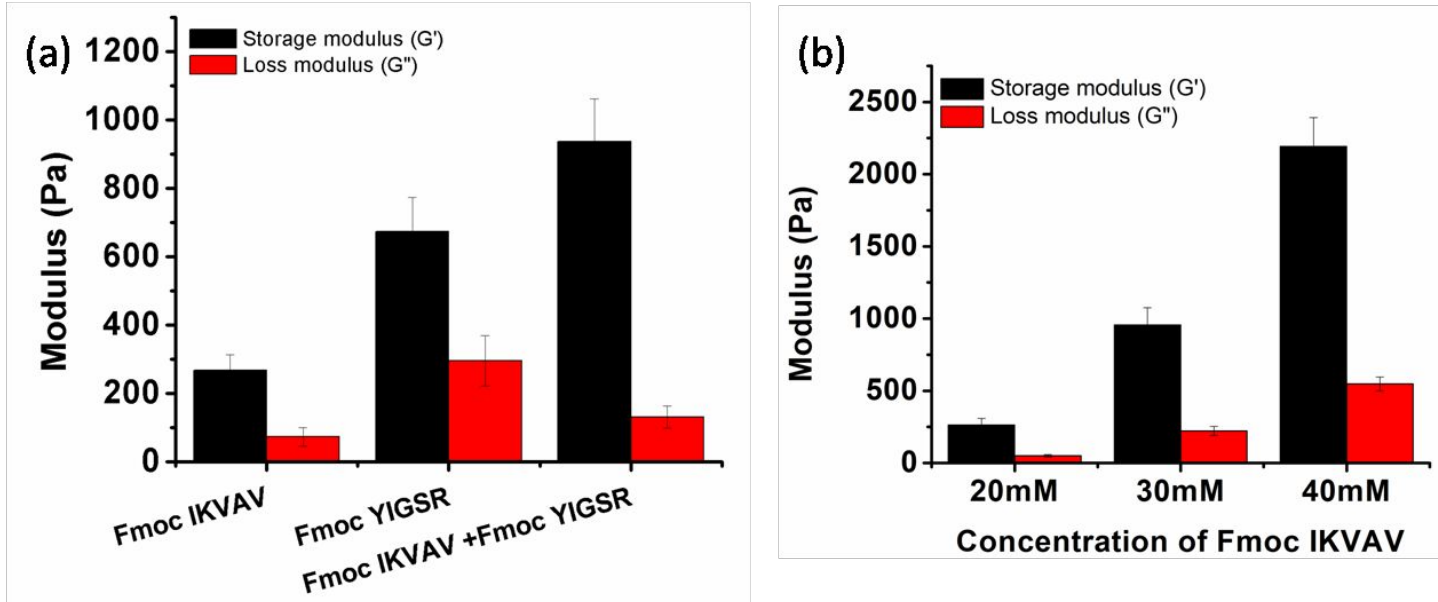

(c)
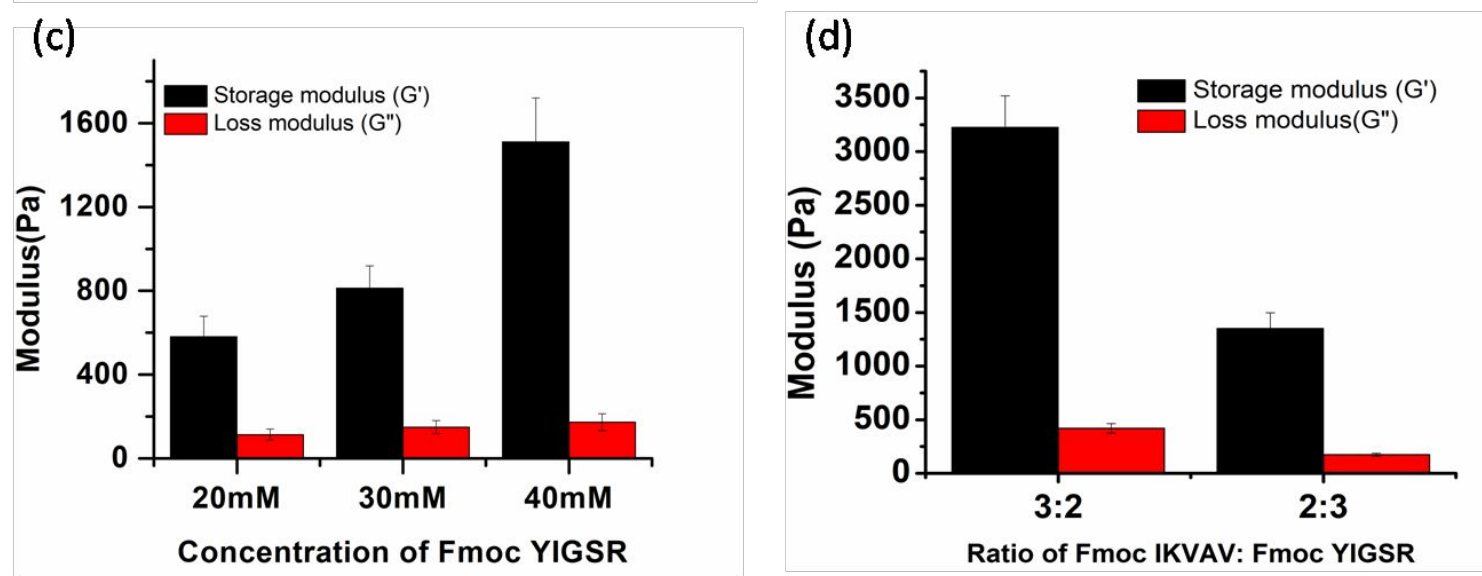

Figure S7: Rheological measurements of laminin derived peptide hydrogels with (a) bar graphs showing the storage and loss modulus of individual and composite peptide hydrogels, (b) concentration dependent rheology of Fmoc IKVAV, (c) concentration dependent rheology of Fmoc YIGSR and (d) composite gels with variable ratios of Fmoc IKVAV and Fmoc YIGSR. 


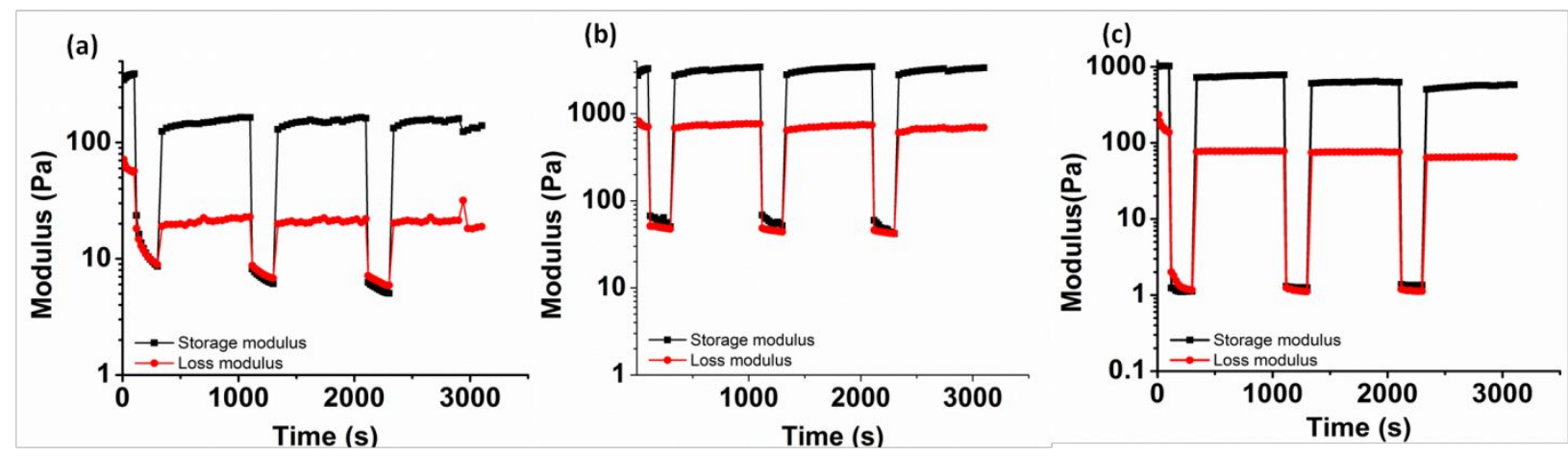

Figure S8: Rheological analysis depicting thixotropic nature of laminin derived peptide hydrogels at a concentration of 20mM of (a) Fmoc IKVAV, (b) Fmoc YIGSR and (c) Fmoc IKVAV +Fmoc YIGSR in 1: 1 molar ratio (10mM each) measured upto 3 consecutive cycles with deformation strain of $50 \%$ at frequency of $1 \mathrm{~Hz}$. 

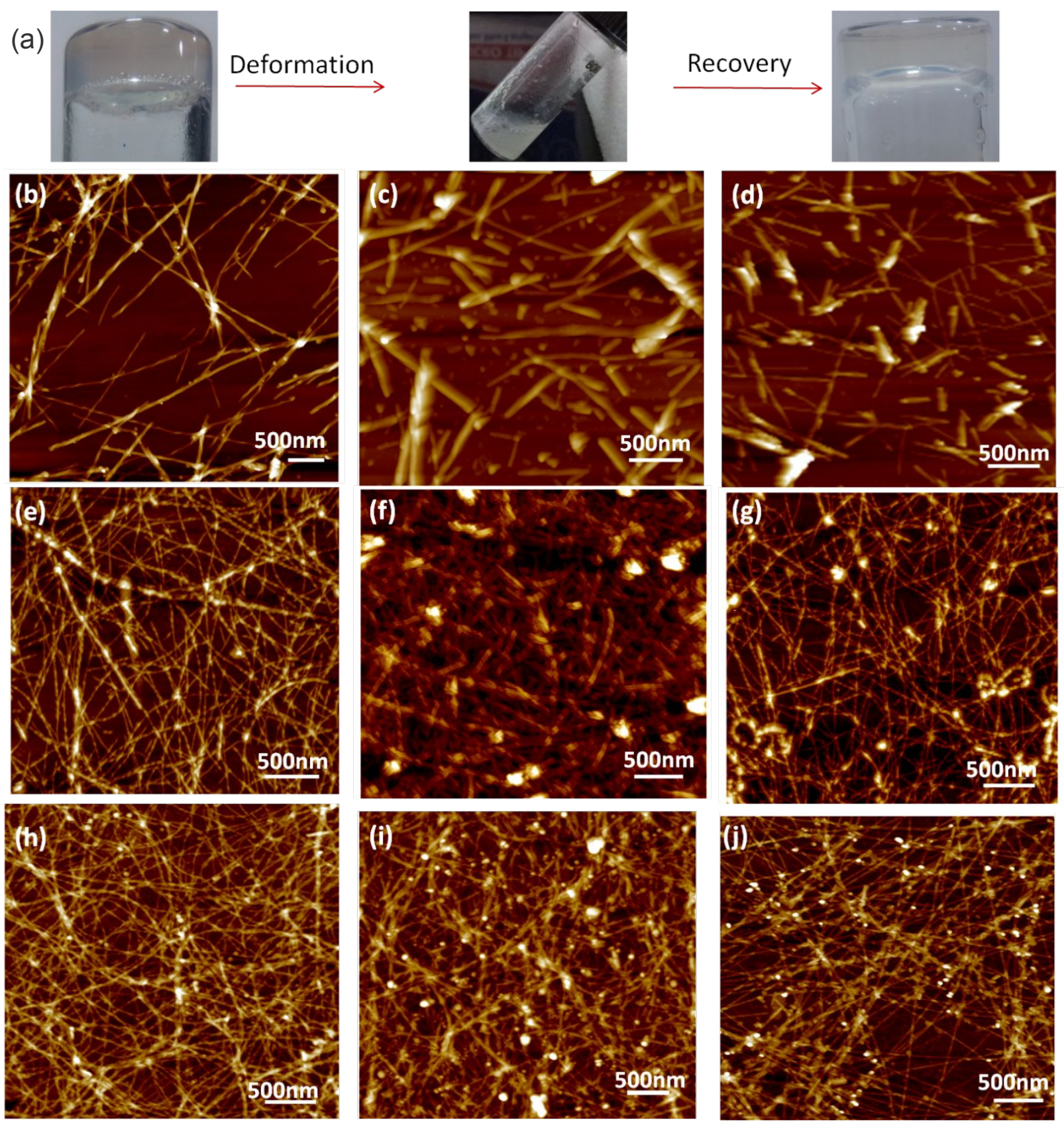

Figure S9: Thixotropic measurements showing gel-sol-gel transformation after applying stress for $5 \mathrm{~min}$ and recovery after $3 \mathrm{hr}$. (a) Optical images of the macroscopic gel and sol transformations. AFM images of (b-d) Fmoc IKVAV, (e-g) Fmoc YIGSR and (h-j) Fmoc IKVAV +Fmoc YIGSR gels (20mM concentration) at different stages of thixotropy measurement i.e. before deformation, after deformation and after 3 hrs of recovery. 

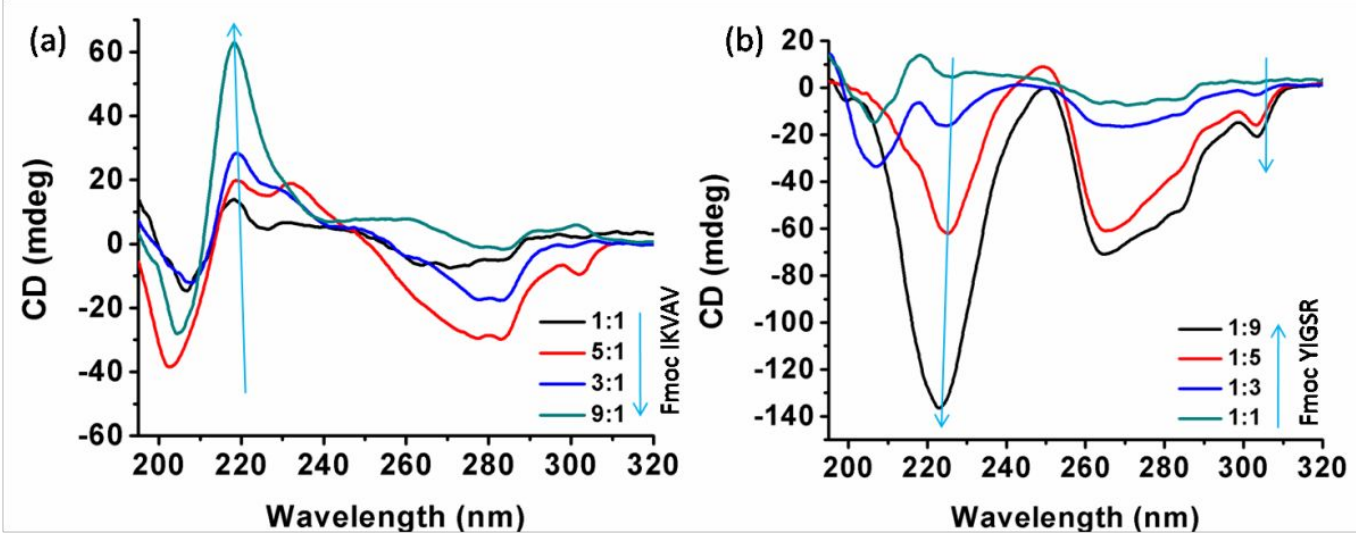

Figure S10: CD signals of composite laminin peptide based gels $(20 \mathrm{mM})$ prepared at different ratios of Fmoc IKVAV and Fmoc YIGSR with (a) increasing concentration of Fmoc IKVAV and (b) increasing concentration of Fmoc YIGSR. 

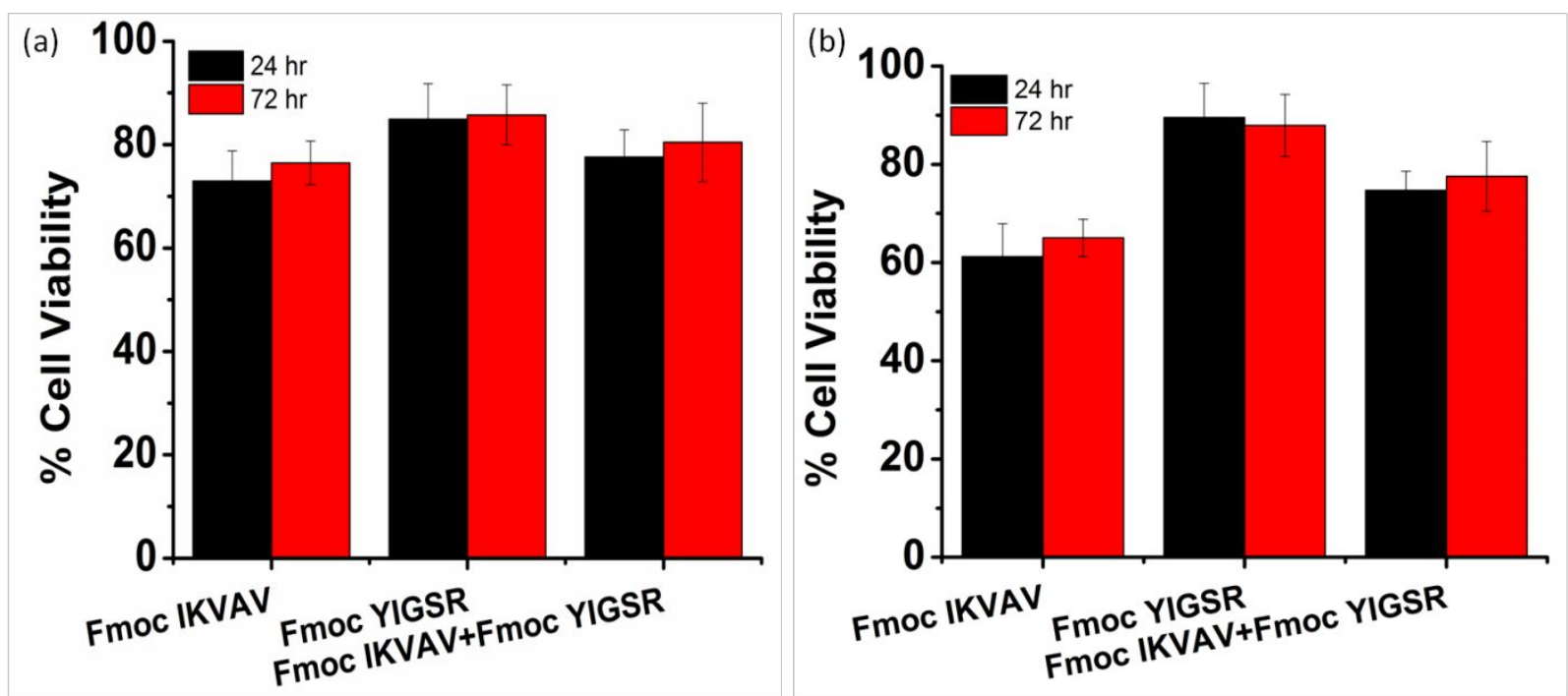

Figure S11: Cytotoxicity studies through MTT assay with (a) C6 cells and (b) SHSY5Y cells after $24 \mathrm{hrs}$ and $72 \mathrm{hrs}$ of culture over laminin derived peptide hydrogels and their conjugate hydrogels. 


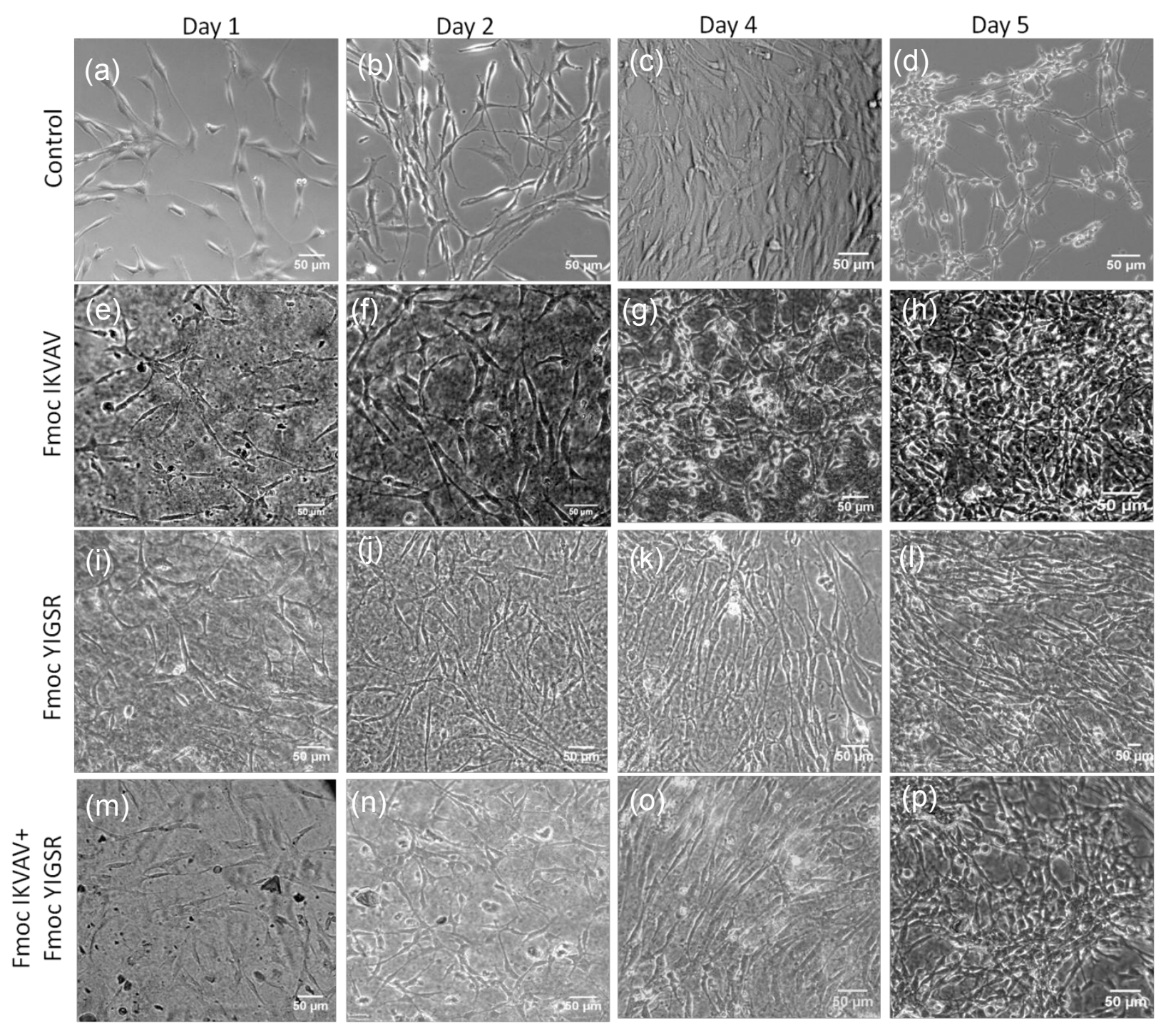

Figure S12: Microscopic images of C6 cells cultured over (a-d) Control (TCPS) and laminin derived peptide hydrogels (e-h) Fmoc IKVAV; (i-l) Fmoc YIGSR and (m-p) Fmoc IKVAV+ Fmoc-YIGSR, at day 1, day 2, day 4 and day5. 
Day 1
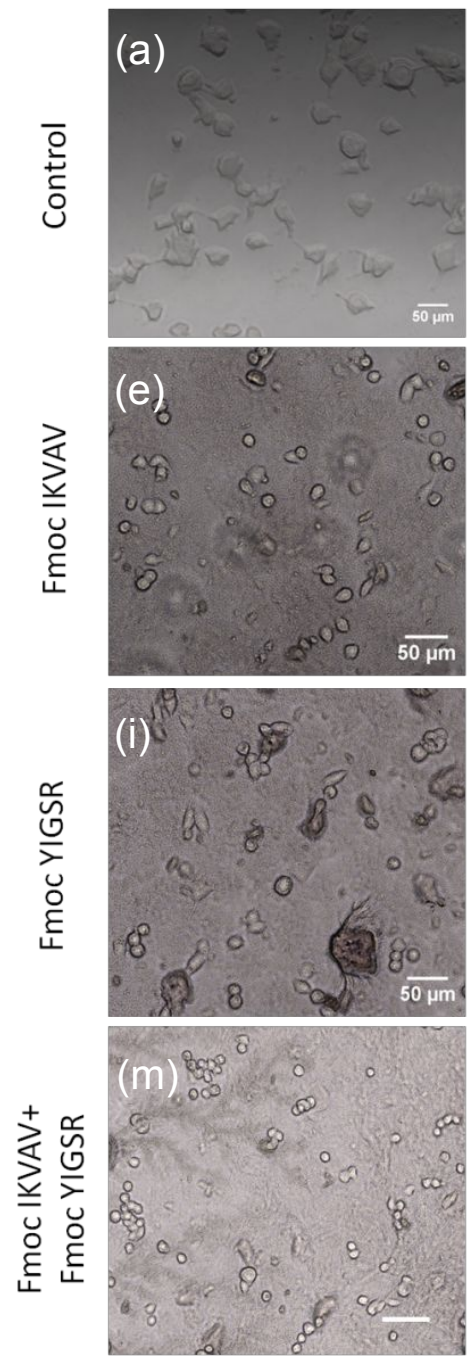

Day 2
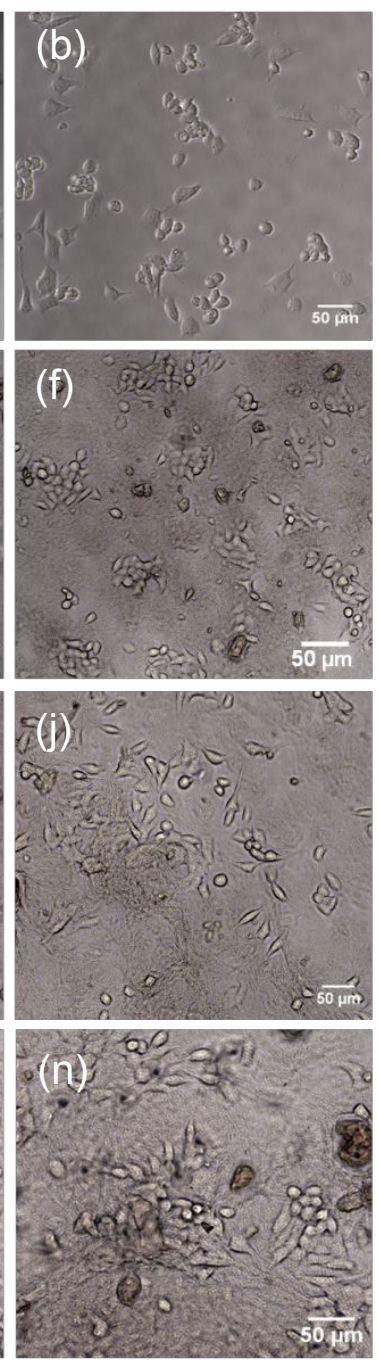

Day 4
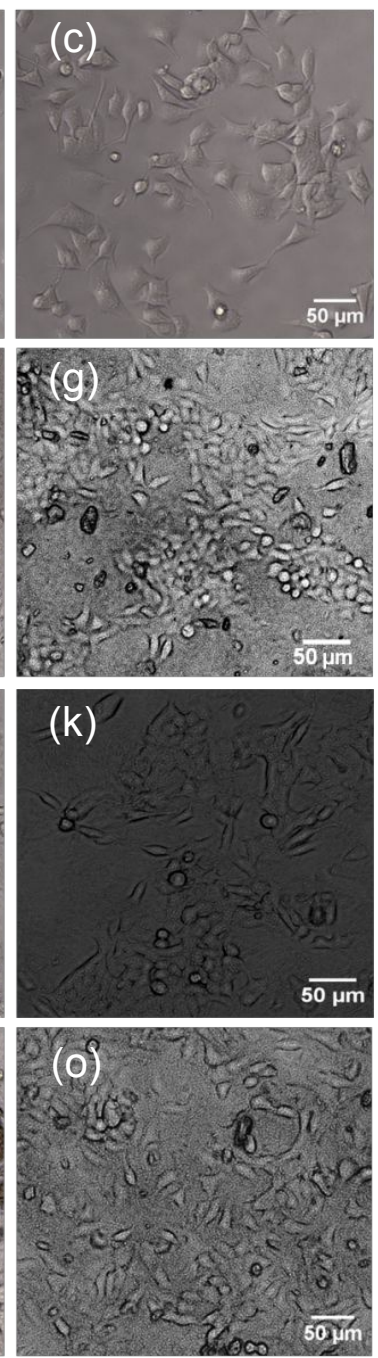

Day 5
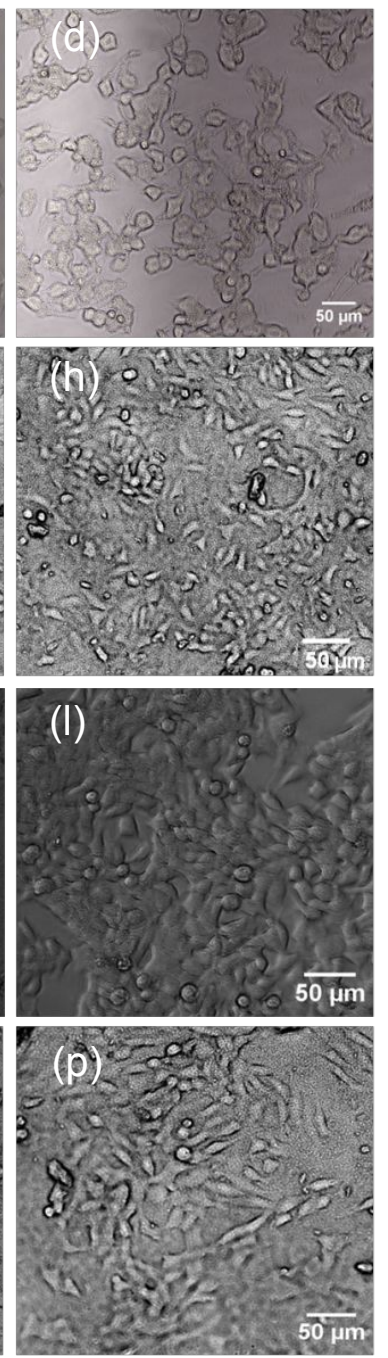

Figure S13: Microscopic images of SHSY5Y cells cultured over (a-d) Control (TCPS) and laminin derived peptide hydrogels (e-h) Fmoc IKVAV; (i-l) Fmoc YIGSR and (m-p) Fmoc IKVAV+ Fmoc-YIGSR, at day 1, day 2, day 4 and day5. 

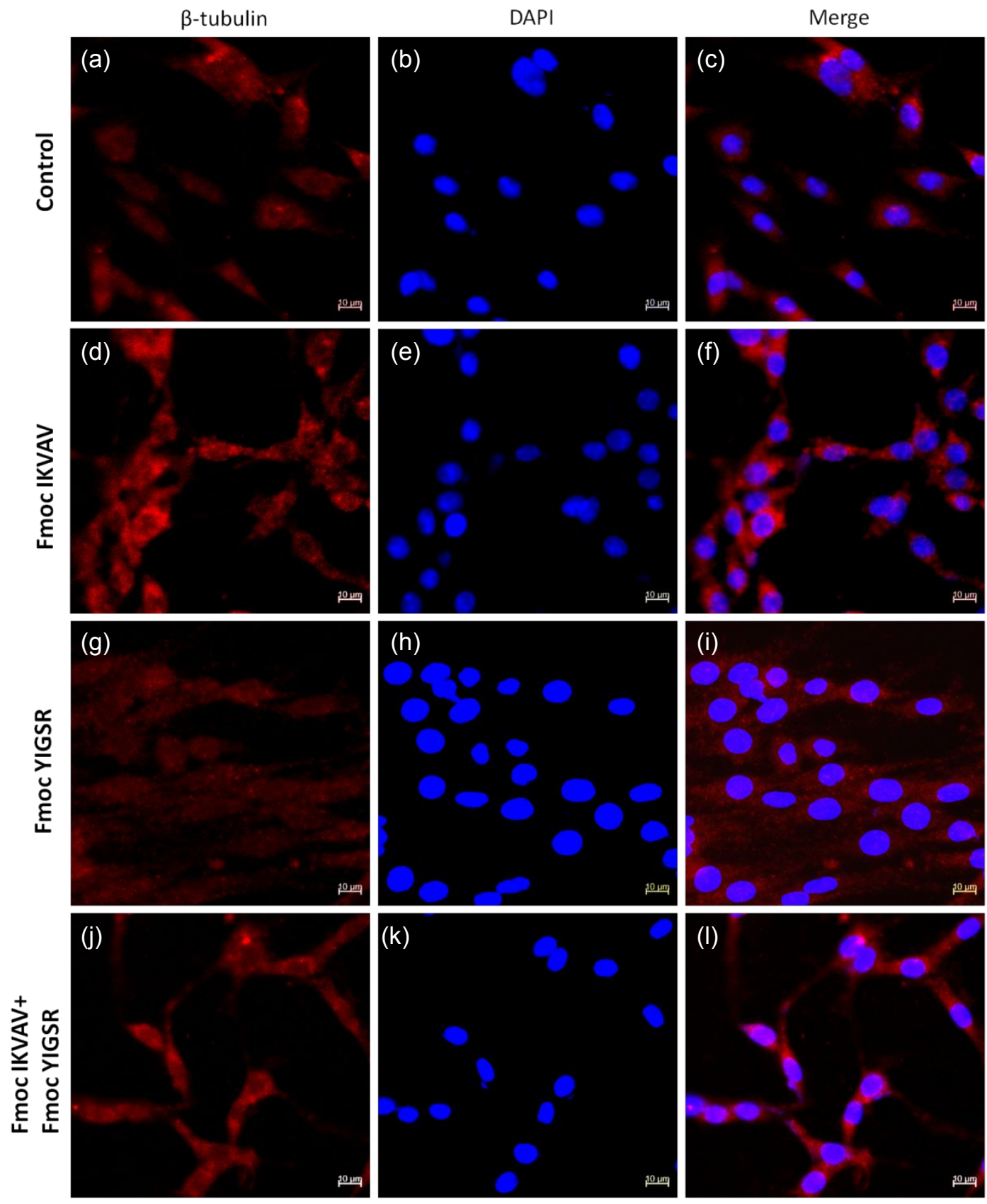

Figure S14: Immunofluorescence staining of neuronal marker $\beta$-III tubulin expressed in C6 cells cultured over (a-c) Control (TCPS), (d-f) Fmoc IKVAV hydrogels, (g-i) Fmoc YIGSR hydrogels and (j-1) conjugate Fmoc IKVAV and Fmoc YIGSR hydrogels, after 5 days. The red colour indicates the $\beta$-III tubulin stained with Alexa Fluor 555 and blue indicates nuclei stained with DAPI. 


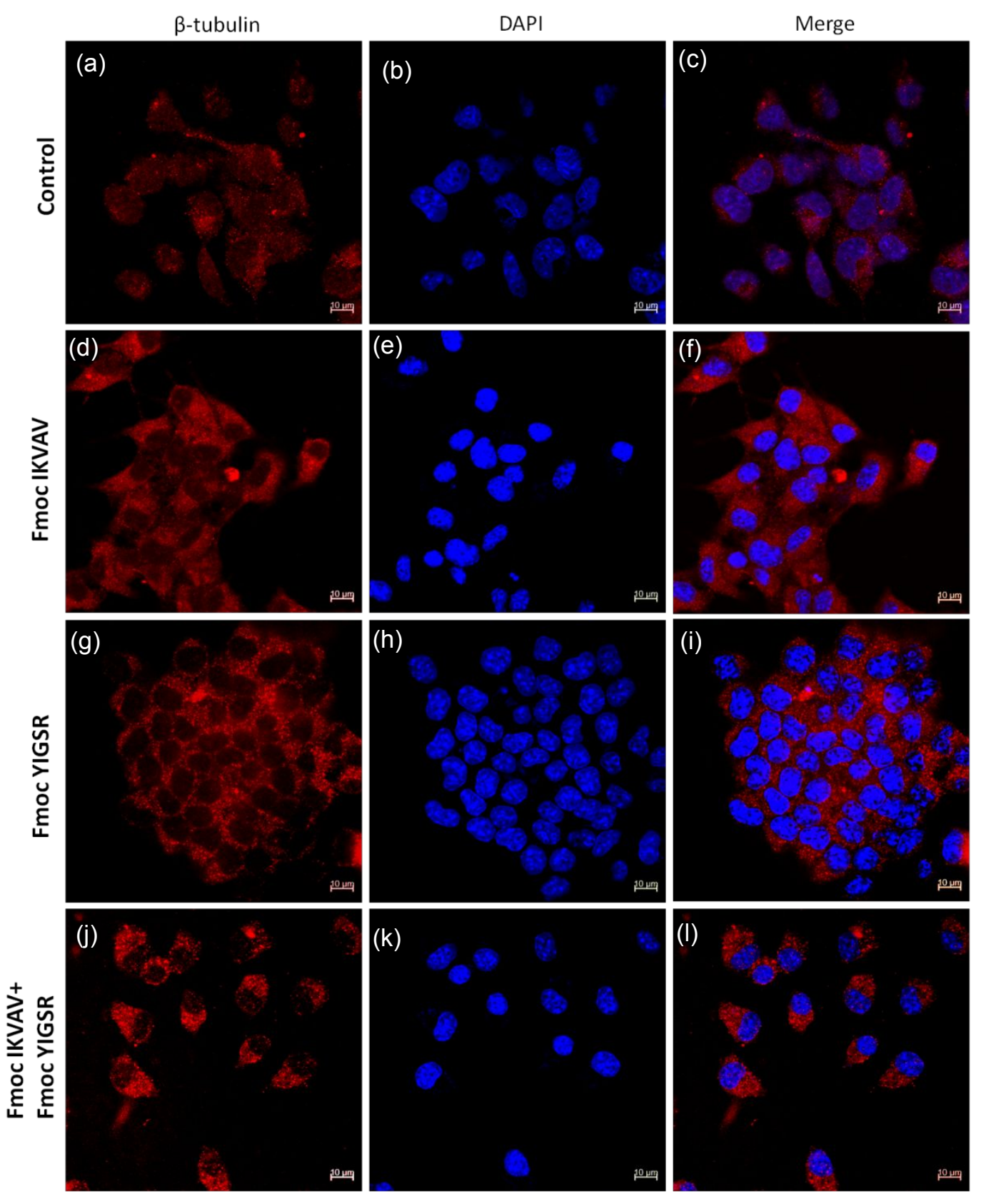

Figure S15: Immunofluorescence staining of neuronal marker $\beta$-III tubulin expressed in SHSY5Y cells cultured over (a-c) Control (TCPS), (d-f) Fmoc IKVAV hydrogels, (g-i) Fmoc YIGSR hydrogels and (j-1) conjugate Fmoc IKVAV and Fmoc YIGSR hydrogels, after 5 days. The red colour indicates the $\beta$-III tubulin stained with Alexa Fluor 555 and blue indicates nuclei stained with DAPI. 

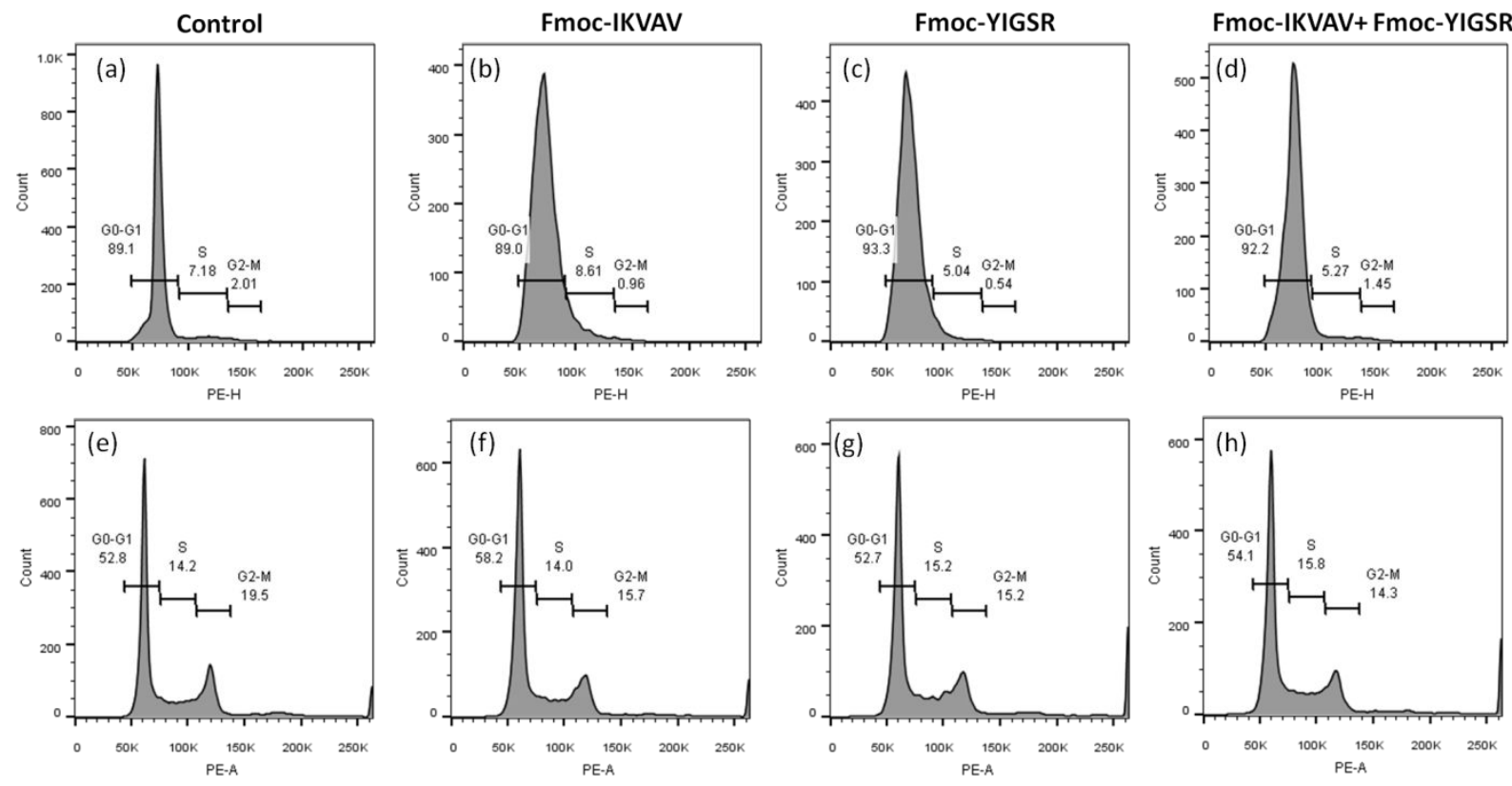

(i)

(j)
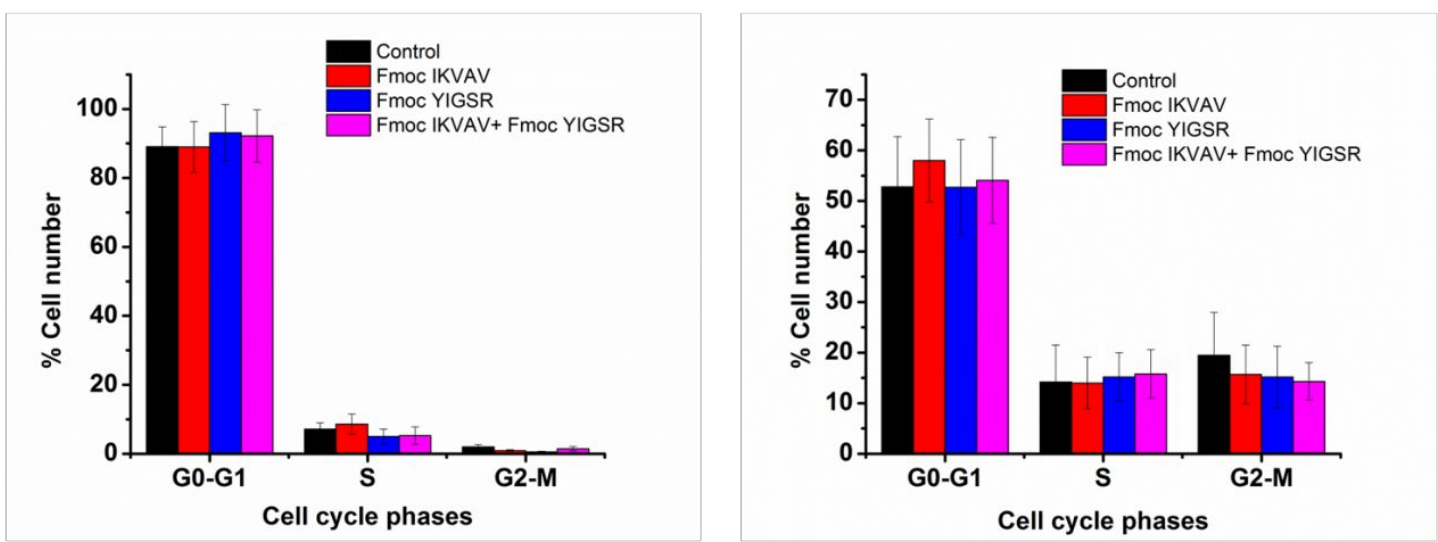

Figure S16: Flow cytometry analysis of cell cycle in (a-d) C6 cells and (e-h) SHSY5Y cells, after the treatment with different laminin peptides hydrogels diluted upto the concentration of $1000 \mu \mathrm{g} / \mathrm{ml}$, after $24 \mathrm{hrs}$. The percentage of cells present in different phases of cell cycle in (i) C6 cells and (j) SHSY5Y cells. 